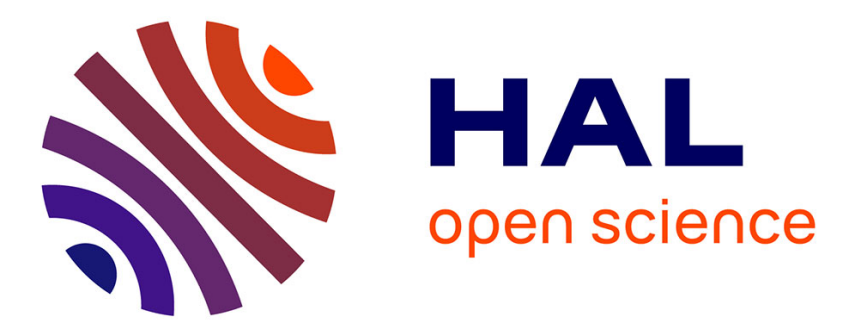

\title{
Spin filtering effect on secondary electrons crossing an iron overlayer
}

\author{
F. Sirotti, G. Panaccione, G. Rossi
}

\section{To cite this version:}

F. Sirotti, G. Panaccione, G. Rossi. Spin filtering effect on secondary electrons crossing an iron overlayer. Journal de Physique IV Proceedings, 1994, 04 (C9), pp.C9-175-C9-178. 10.1051/jp4:1994929 . jpa-00253490

\section{HAL Id: jpa-00253490 https://hal.science/jpa-00253490}

Submitted on 1 Jan 1994

HAL is a multi-disciplinary open access archive for the deposit and dissemination of scientific research documents, whether they are published or not. The documents may come from teaching and research institutions in France or abroad, or from public or private research centers.
L'archive ouverte pluridisciplinaire HAL, est destinée au dépôt et à la diffusion de documents scientifiques de niveau recherche, publiés ou non, émanant des établissements d'enseignement et de recherche français ou étrangers, des laboratoires publics ou privés. 


\title{
Spin filtering effect on secondary electrons crossing an iron overlayer
}

\author{
F. Sirotti*,**, G. Panaccione ${ }^{* *}$ and G. Rossi*,** \\ * Laboratorium für Festkörperphysik, ETH-Zurich, 8093 Zurich, Switzerland \\ ** Laboratoire pour l'Utilisation du Rayonnement Electromagnétique, CNRS-CEA-MEN, 91405 Orsay, \\ France
}

\begin{abstract}
Spin polarization (SP) measurements of the secondary electron yield from ferromagnetic interfaces is a sensitive probe of surface and interface magnetism. By exploiting the jumps of the total photoionization cross section in correspondence of the L-edge excitation in transition metals one introduces a chemical sensitivity in the SP via the enhancement of the secondary emission from a specific magnetic or non magnetic specie at the interface. The $\mathrm{h} \nu$-dependence of the SP in overlayer experiments allows to derive accurately the total and spin-dependent attenuation lengths for secondary electrons in non-magnetic and ferromagnetic materials.
\end{abstract}

The energy dependence of the electron transport in solids is a central issue for the understanding of electron spectroscopies[1] Inelastic electron-electron scattering is assumed to dominate the electron transport in solids and near the surface. In recent overlayer experiments the measurement of the spin polarization (SP) of the ejected secondary electrons has provided evidence of large deviations from the "universal curve" of the inelastic mean-free path at low electron kinetic energies, at least for ferromagnetic solids [2,3]. Furthermore clear evidence for a spin- dependence of the electron mean-free path in ferromagnetic solids has been obtained from photoemission[4] and from tunneling measurements [5], and it has been interpreted in connection with the structure of empty d-band states. $[6,7] \mathrm{A}$ Fe-thickness dependent oscillatory period of the perpendicular magnetoresistance in $\mathrm{Fe} / \mathrm{Cr}$ multilayers has been explained by spin barriers for electron transport. [8]

We present here a new kind of overlayer experiment, which is based on the exploitation of the photon-energy dependent photoionization cross sections and on the tunability of synchrotron radiation: we have measured the SP of the (partial) secondary-electron yield as excited mostly in the substrate, or in the spacer layer, or in the overlayer in $\mathrm{Fe}(100) / \mathrm{Cr}$ and $\mathrm{Fe}(100) / \mathrm{Cr} / \mathrm{Fe}$ interfaces. By tuning the photon energy to the characteristic $\mathrm{L}_{2,3}$ edges of $\mathrm{Cr}$ (or $\mathrm{Fe}$ ) the relative contribution of the $\mathrm{Cr}(\mathrm{Fe})$ layers to the total signal is increased by a factor 5 and consequently the total SP changes. In all cases the ejected electron energies can be defined at a wanted value by the detection system. With this technique one can have for the same overlayer thickness at least two independent measures of SP which depend on the localization of the primary electron (photoelectrons and Auger electrons) source and on the different depths of material traversed by the secondary electrons. We present here results for the attenuation of the SP of the $\mathrm{Fe}(100)$ secondary yield through $\mathrm{Cr}$ overlayers, and for a $\mathrm{Fe} / \mathrm{Cr} / \mathrm{Fe}$ sandwich. The ejected electrons were roughly selected in energy by a multiple einsel lens system and the SP of the partial yield was measured, after acceleration to $100 \mathrm{KeV}$, by Mott scattering on a $A u$ foil. The SP is defined as $P=\left(N^{\uparrow}-N^{\downarrow}\right) /\left(N^{\dagger}+N^{\downarrow}\right)$ where $N^{\uparrow}\left(N^{\downarrow}\right)$ is the number of electrons with magnetic moment parallel (antiparallel) to the sample magnetization $M$. Data were collected for photon energies giving roughly the same sensitivity to $\mathrm{Fe}$ and $\mathrm{Cr}$, or, at the L-edge en- 
ergies where a large enhancement factor in the absorption determines a "localization" of the excited electron source either in the Cr layer or in the Fe substrate (and overlayer). The simultaneous fit of the SP vs. thickness curves determines the two inelastic mean free path $(\lambda)$ values for majority and minority electrons.
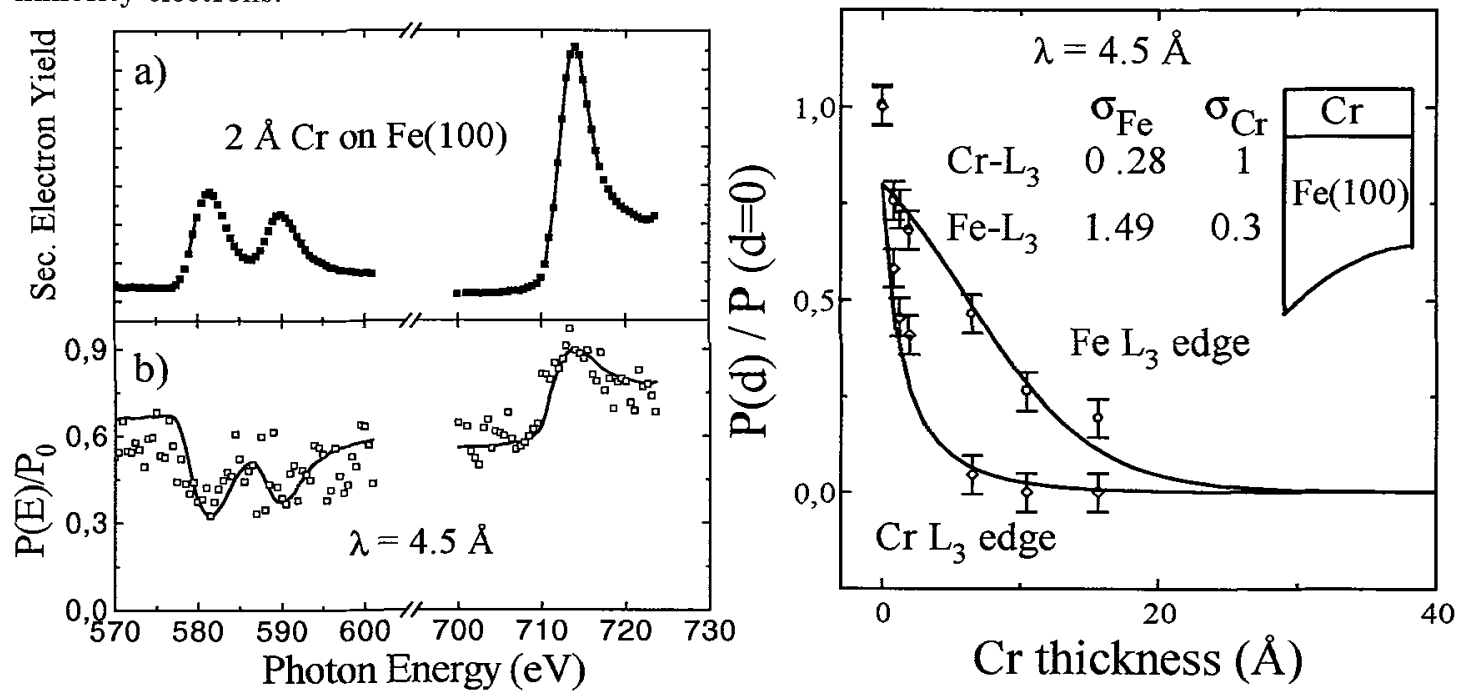

Fig.1: Photon energy dependence of (a) the total electron yield near the $L$ absorption edges of $\mathrm{Cr}$ and $\mathrm{Fe}$; (b) the secondary electron SP

Fig.2: Attenuation of the $\mathrm{Fe}(100) \mathrm{SP}$ as a function of the epitaxial Cr overlayer thickness measured at the L-edges of $\mathrm{Cr}$ and Fe.

The experiments were performed in a UHV spectrometer with base pressure lower then $1 \cdot 10^{-10}$ mbar following surface science methods on a $\mathrm{Fe}-3 \% \mathrm{Si}[100]$ oriented single crystal substrate mounted on a horseshoe soft iron yoke.[9] The typical value of SP measured at remanence for a clean $\mathrm{Fe}(100)$ surface is $32 \%$, i.e. close to the actual band polarization of Fe. Photoemission spectroscopy was used as a surface diagnostic of coverage, cleanliness and magnetic order via the measure of the linear magnetic dichroism in the angular distribution of the photoelectrons (LMDAD).[10]

The photon energy dependence of the photoejected yield is shown in figure $1 \mathrm{a}$ in the energy region of the $\mathrm{L}_{2,3}$ edges of $\mathrm{Cr}$ and $\mathrm{Fe}$, for $2 \AA \mathrm{Cr}$ epitaxially grown on $\mathrm{Fe}(100)$. The large increase of the yield in correspondence of the $\mathrm{L}_{2,3}$ excitation is due to the large cross section for $2 \mathrm{p}-3 \mathrm{~d}$ dipole transitions, which enhances by a factor 5 the contribution of the excited atoms. Simultaneously to the absorption measurement the SP analysis is done. The energy dependence of the SP is represented in fig $1 \mathrm{~b}$. At all photon energies the measured SP is due to the Fe substrate attenuated by the unpolarized yield from the $\mathrm{Cr}$ overlayer. As the X-ray absorption cross section of $\mathrm{Cr}(\mathrm{Fe})$ increases at the $\mathrm{L}_{2,3}$ edges the measured SP decreases (increases), due to the enhanced contribution of unpolarized (polarized) electrons originating from $\mathrm{Cr}(\mathrm{Fe})$. The continuous line through the experimental spin polarization data points is obtained by the following expression

$$
\frac{P}{P_{F e}}=\frac{\sigma_{F e} \cdot e^{-\frac{d}{\lambda}}}{\sigma_{F e} \cdot e^{-\frac{d}{\lambda}}+\sigma_{C r} \cdot\left(1-e^{-\frac{d}{\lambda}}\right)}
$$

where $\mathrm{P}_{F_{e}}$ is the SP measured on the clean Fe surface, $\mathrm{d}$ is the film thickness in $\AA$, and $\sigma_{F_{e}}$, $\sigma_{C r}$ are the photoionization cross sections of $\mathrm{Fe}$ and $\mathrm{Cr}$ which are the measured absorption spectra normalized to the tabulated cross sections values [11] below the edges, $\lambda$ is the attenuation length of the iron signal through chromium. In Fig. 2 the measured SP is reported as a function of the $\mathrm{Cr}$ coverage. Every point represents the remanent SP of the partial ejected electron yield measured with $\mathrm{h} \nu=710 \mathrm{eV}$, i.e. at the $\mathrm{L}_{3}$ edge of $\mathrm{Fe}$ or with $\mathrm{h} \nu=582 \mathrm{eV}$, i.e the $\mathrm{L}_{3}$ edge of $\mathrm{Cr}$. The continuous lines are obtained with (1) for a value of $\lambda=4.5 \AA$. 
Values of 3.7+- 0.05 Afor the Fe SP attenuation through $\mathrm{Cr}[3]$ or Ta [2] were obtained in electron excited experiments in the approximation of identical secondary yield for substrate and overlayer. The clear advantage of our synchrotron radiation experiment is the chemical sensitivity which adds a constrain to the determination of the inelastic mean free path from overlayer data.

By depositing $\mathrm{Fe}$ onto the $\mathrm{Cr}(100)$ surface of an epitaxial $5 \mathrm{ML} \mathrm{Cr} / \mathrm{Fe}(100)$ sample, one grows a new epitaxial overlayer antiferromagnetically coupled to the Fe substrate. $[12,13]$ The magnetization direction of the iron overlayer was checked using the Linear Magnetic Dichroism in the Angular Distribution of photoelectrons (LMDAD) [9].

The magnetic hysteresis loops for two Fe thicknesses $(1.8 \AA$ and $3.8 \AA)$ on $5 \mathrm{ML} \mathrm{Cr} / \mathrm{Fe}(100)$ are reported in Fig. 3. The SP was measured for a photoelectric yield excited at the L-edge energies of $\mathrm{Cr}$ and $\mathrm{Fe}$. The substrate magnetization dominates the SP hysteresis for the thinnest Fe overlayer when measured with $\mathrm{h} \nu=710 \mathrm{eV}$, but a reversed hysteresis loop, indicating antiferromagnetism of the overlayer is measured with $\mathrm{h} \nu=582 \mathrm{eV}$. At the $\mathrm{Cr}$ edge energy most of the signal originates in the spacer layer which is unpolarized (in average): the SP which is measured is therefore due to the weak contribution of the overlayer, and to the spin filtering effect suffered by the electrons originated in the Cr layer and scattered by the ferromagnetic overlayer. For double overlayer thickness (Fig. $3 \mathrm{c}, \mathrm{d})$ the SP opposite to the substrate magnetization direction is large at the Cr edge, and small at the $\mathrm{Fe}_{3}$ edge due to averaging of the $\mathrm{Fe}$ substrate and $\mathrm{Fe}$ overlayer signals.

The dependence of SP upon the Fe overlayer thickness is analyzed in figure 4. It is not possible to explain these results with formula 1. In the three-layer structure the contributions to the total spin polarized signal come from the $F e$ substrate $\mathrm{I}_{F e}$, from the chromium interlayer $\mathrm{I}_{C r}$ and from the iron overlayer $\mathrm{I}_{O L}$; by introducing independent attenuation lengths for the majority $(+)$ and the minority (-) electrons, in the reference frame of the substrate iron, the ejected majority electrons originating from the three regions can be written as

$$
\begin{aligned}
& I_{F e}^{+}=N^{+} \sigma_{F e} e^{-\frac{d}{\lambda_{C r}}} \cdot e^{-\frac{d}{\lambda^{-}}} \\
& I_{C r}^{+}=\frac{1}{2} \cdot \sigma_{C r} \cdot\left(1-e^{-\frac{d_{C r}}{\lambda_{C r}}}\right) \cdot e^{-\frac{d}{\lambda^{-}}} \\
& I_{O L}^{+}=N^{-} \cdot \sigma_{F e} \cdot\left(1-e^{-\frac{d}{\lambda^{-}}}\right)
\end{aligned}
$$

where $\mathrm{N}^{+}\left(\mathrm{N}^{-}\right)$indicates the number of majority (minority) electrons emitted from iron and can be determined from the SP measured on the clean iron surface, $\mathrm{d}_{C r}$ and $\mathrm{d}$ are the thicknesses of the chromium and iron layers respectively, $\lambda_{C r}$ is the attenuation length in chromium, $\lambda^{+}\left(\lambda^{-}\right)$is the spin dependent attenuation length of majority (minority) electrons in the Fe overlayer. By reversing + and - signs the minority spin electron currents can be written.

If the SP is given by $p=\left(I^{+}-I^{-}\right) /\left(I^{+}+I^{-}\right)$the above formulation allows to fit the two sets of experimental data points having as free parameters only the two spin dependent attenuation lengths. The resulting dependence from the Fe overlayer thickness is represented from the continuous lines in Fig. 5 which are obtained for $\lambda^{+}=6.5$ Åand $\lambda^{-}=5.2 \AA$.

The accuracy of the results depends on the accuracy of the thickness calibration of the experiment. The relative cross section values can be measured accurately on standards of the two materials, and do not introduce systematic errors in the derivation of the total and spin-dependent inelastic mean free paths. Our results confirm the small value of $\lambda$ for secondary electrons in $\mathrm{Cr}$, and show that the spin filtering effect in $\mathrm{Fe}$ amounts to a $20 \%$ difference of the mean free paths for secondary electrons. This result is in good agreement with the experiment of Pappas et al. who measured the SP of the $\mathrm{Cu} 3 \mathrm{~d}$ photoemission filtered by a ferromagnetically ordered Fe overlayer.[4] The absolute values of $\lambda^{+}$and $\lambda^{-}$are also in agreement considering the different densities of $\mathrm{Fe}(100)$ planes epitaxially grown on $\mathrm{Cr}(100)$ and $\mathrm{Cu}(100)$, and the accuracy of thickness calibration. [4] We have shown that the analysis of the ferromagnetic or antiferromagnetic coupling of ultrathin overlayers to the substrate can be made much more sensitive by exploiting the "localization" effect of the internal source of primary electrons in the substrate, or interlayer, or overlayer which is obtained by exploiting the jumps of the photoionization cross section for a given element at characteristic photon energies.

The spin filtering effect has important implications. 1) the evaluation of spin polarized spectroscopies and of surface magnetometry based on SP measurements of low energy electrons must take 
into account spin filtering; 2) spin resolved data from interfaces must be evaluated by taking into account spin filtering; 3) the derived characteristic lengths of $\lambda^{+}=6.5 \AA$ and $\lambda^{-}=5.2 \AA$ for secondary electrons allow the design of electron spin analyzers based on (quasi)monochromatic electron beams [7] with an efficiency of some percents (the efficiency of high energy Mott scattering detectors is $\left.10^{-4}\right)$.

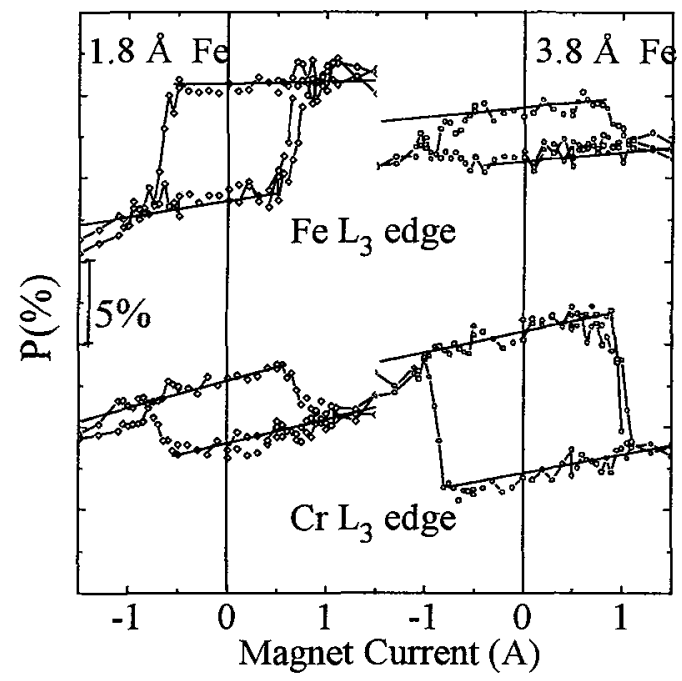

Fig.3: Magnetization (SP) hysteresis loops of $1.8 \AA \mathrm{Fe}$ and $3.8 \AA \mathrm{Fe}$ overlayers on $5 \mathrm{Ml} \mathrm{Cr} / \mathrm{Fe}(100)$ measured at the $\mathrm{L}$ edges of $\mathrm{Fe}$ and $\mathrm{Cr}$.

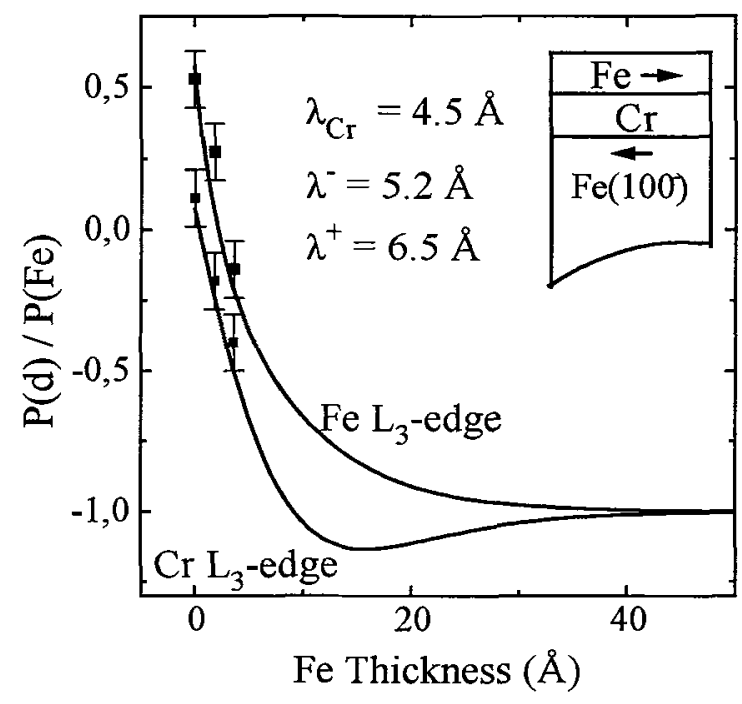

Fig.4: Variation of the SP of secondary electrons in the $\mathrm{Fe} / \mathrm{Cr} / \mathrm{Fe}(100)$ system as a function of Fe overlayer thickness.

This work was supported by the Swiss National Fund under Program 24 and by the EC Human Capital and Mobility Program.

\section{References:}

[1] M.P.Seah, W.A.Dench, Surf. Interface Anal. 1,2 (1979).

[2] O.Paul, S.Toscano, K Totland, M.Landolt, Surf. Sci. 251/252, 27 (1991);

O.Paul Dissertation ETH Zurich, No 9210 (1990).

[3] M.Donath, D.Scholl, D.Mauri, E.Kay, Phys. Rev. B 43,16 (1991) 13164.

[4] D.P.Pappas, K.P. Kämper, B.P. Miller, H.Hopster, D.E. Fowler, C.R. Brundle, A.C.Luntz, Z .X. Shen Phys. Rev. Lett. 66, 504 (1991).

[5] B.A. Gurney, V.S. Speriosu, J.P. Nozieres, H. Lefakis, D.R. Wilhoit, O.U. Need,

Phys. Rev. Lett. 71, 4023 (1993).

[6] H.C. Siegmann, J.Phys.Condens.Matter 4 (1992) 8395.

[7] G.Schonhense, H.C. Siegmann, Ann. Physik 2 (1993) 465.

[8] S.N. Okuno, K. Inomata, Phys. Rev. Lett. 72,10 (1994) 1553.

[9] F.Sirotti, G.Rossi, Phys. Rev. B 49 (1994).under press

[10] G.Rossi, F.Sirotti, N.A. Cherepkov, F.Combet Farnout, G.Panaccione,

Solid State Comm. 90 (1994) 557.

[11] J.J. Yeh, I. Lindau, At. Data Nucl. Data Tables 32,(1985) 1.

[12] J.Unguris, R.J.Celotta, D.T.Pierce, Phys. Rev. Lett. 69, (1992).

[13] C. Turtur, G. Bayreuther, Phys. Rev. Lett. 72, 10 (1994) 1557. 